\title{
Recombinant Factor VIIa for Uncontrolled Bleeding in 2 Different Cases of Coagulopathy
}

\author{
Jamal Islam, MD, MS, Syed Azhar, MD, MBA, Jack B. Alperin, MD, and \\ Irshad Syed, $M D$
}

Coagulation factor VIIa (Recombinant) (rF.VIIa; NovoSeven) was approved for use in the United States by the Food and Drug Administration in March 1999 for the treatment of bleeding episodes in patients with hemophilia A or B with inhibitors to factor VIII or factor IX. Off-label use of rF.VIIa to control uncontrolled bleeding in different clinical scenarios has been reported in the literature.

Recombinant factor VIIa (rF.VIIa) has been used to control refractory bleeding peptic ulcer. ${ }^{1}$ It has also been used for uncontrolled bleeding after bowel surgery in patients suffering from Crohn disease and large bowel lymphoma with no previous history of coagulopathy. ${ }^{2}$ Patients with liver cirrhosis and coagulopathy who were given rF.VIIa avoided bleeding complications when their liver biopsies were performed. ${ }^{3}$ Administration of $\mathrm{rF}$ .VIIa controls the bleeding in trauma-related multitransfusion dilutional coagulopathy. ${ }^{4,5}$ It also stops uncontrolled nasal and dental bleeding in patients on warfarin. ${ }^{6}$

We report our success in attaining hemostasis with the use of rF.VIIa in 2 patients having uncontrolled bleeding associated with liver cirrhosis and warfarin toxicity. Our experience includes further information on the use of rFVIIa in uncontrolled bleeding and may provide grounds for further investigation to extend the indication of rFVIIa. This will greatly enhance our capability to save lives in desperate situations.

Submitted, revised, May 11, 2003.

From the Department of Family Medicine (JI, SA, IS) and Department of Internal Medicine, Division of Hematology/ Oncology (JBA), University of Texas Medical Branch, Galveston. Address correspondence to Jamal Islam, MD, MS, Department of Family Medicine, University of Texas Medical Branch, 301 University Boulevard, Galveston, TX 77555-1123 (email: jaislam@utmb.edu).

\section{Case Report 1 \\ Presenting Complaint}

A 51-year-old woman was admitted on March 24, 2001, complaining of left leg swelling and pain. No trauma was reported. She was noted to have coagulopathy, with a prothrombin time (PT) of 17.8 seconds and activated partial thromboplastin time (aPTT) of 36 seconds.

\section{Past Medical History}

The patient had a history of alcohol abuse, hepatitis $\mathrm{C}$, and liver cirrhosis. She had been discharged from the hospital on January 6, 2001, after 10 days hospitalization for anemia and left lower leg swelling. The magnetic resonance imaging and magnetic resonance angiography of the swollen leg showed an $18 \times 10 \times 9$-cm mass in the left calf. It was fairly well marginated, septated, and located between the gastrocnemius and soleus muscles. There was a suggestion of a hemorrhagic or highly proteinaceous component. An ultrasound-guided drainage was conducted, and approximately 800 $\mathrm{mL}$ of blood was removed. A 10 French catheter was placed for continuous drainage. Analysis of the fluid was negative for any bacterial growth. A follow-up sonogram of the left calf performed the next day showed good resolution of the mass with a residual $30 \mathrm{~mL}$ of fluid noted. During the 10 days of her hospital stay, she received 7 units of packed red blood cells because of dropping hemoglobin (Hb) and hematocrit (Hct) levels. Except for the intramuscular bleeding, no other causes were detected. Upon discharge from the hospital, her $\mathrm{Hb}$ and Hct was $9.6 \mathrm{~g} / \mathrm{dL}$ and 0.27 , respectively, platelet count was $61,000 / \mathrm{cm}^{3}$, and PT was $18.8 \mathrm{sec}-$ onds. On March 1, 2001, the catheter was removed from the calf after repeat ultrasound showed only a small amount of fluid.

The patient's admission on March 24, 2001, was driven by the fear that her left leg swelling and pain 
was caused by recurrence of bleeding in her calf. Her laboratory values upon admission were: $\mathrm{Hb}$, $7.9 \mathrm{~g} / \mathrm{dL}$; Hct, 0.238; platelet count, $98,000 / \mathrm{cm}^{3}$; PT, 16.4 seconds; and aPTT, 40 seconds. An ultrasound of her left leg showed collection of fluid in an area measuring of $9.4 \times 2.9 \times 5.5 \mathrm{~cm}$. To exclude arterial malformation in the calf, an arteriogram was performed. This showed the left peroneal artery with large branches coming off in its distribution. There was active hyperemia around the wall of the cavity. This was attributed to the inflammatory process. Because of this procedure (arteriogram), there was oozing of blood from the right groin where the femoral artery was punctured to gain access for the arteriogram. Bleeding could not be stopped by application of pressure over the right groin. Later, cauterization of the site with silver nitrate was performed. The cauterization helped to stop the oozing, but the patient developed a right hip hematoma, which was left to resolve on its own.

On April 4, 2001, the calf swelling was not resolving; therefore, it was aspirated using an 18gauge Chiba needle but produced only $50 \mathrm{~mL}$ of sanguineous fluid. Repeat magnetic resonance imaging was done on April 9, 2001, and the mass measured $17.6 \times 1.8 \times 3.7 \mathrm{~cm}$ (length $\times$ anteroposterior depth $\times$ width). The next day, it was decided to place a catheter for continuous drainage. On April 19, this catheter was removed from the calf after resolution of the hematoma was confirmed by ultrasound.

The patient was found to have guaiac-positive stools on April 15, and esophagogastroduodenoscopy was performed on April 16. A bleeding vessel in the second portion of the duodenum was found and treated with adrenaline injection. On the same day, the patient suffered a fall while visiting the hospital gift shop despite a warning not to ambulate. She suffered a 2-cm laceration to her scalp. She did not report loss of consciousness. Computed tomographic scan of the cranium showed no emergent hemorrhage, mass, or edema. Despite pressure on the scalp, there was continuous oozing from the wound. A surgical team was consulted, and staples were placed to stop the bleeding. On April 20, her $\mathrm{Hb}$ was $10.7 \mathrm{~g} / \mathrm{dL}$, Hct was 0.318 , platelet count was $84,000 / \mathrm{cm}^{3}$, PT was 15.9 seconds, aPTT was 34 seconds, and international normalized ratio (INR) was 1.3. Ten milligrams of vitamin $\mathrm{K}$ was administered subcutaneously. The bleeding from the scalp wound continued; on April 24, the staples were removed and sutures were applied after draining a hematoma under the scalp. On April 26, $\mathrm{Hb}$ and Hct dropped to $7.8 \mathrm{~g} / \mathrm{dL}$ and 0.236 , PT to 10.2 seconds, and aPTT to 38 seconds. A hematology consult was requested at this stage. The decision was made to administer $50 \mu \mathrm{g} / \mathrm{kg} \mathrm{rF}$.VII as a bolus and to repeat in 2 hours if the bleeding continued. The patient stopped bleeding after the first shot of rF.VII. The patient was eventually discharged from the hospital on April 29, 2001, with no further recurrence of bleed from anywhere and was in stable condition. Her laboratory results at time of discharge were: $\mathrm{Hb}, 9.8 \mathrm{~g} / \mathrm{dL}$; Hct, 0.293; platelet

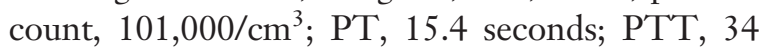
seconds; and INR, 1.3. She no longer complained of her leg swelling or pain. The patient continues to be followed in the clinic for her cirrhosis.

\section{Case Report 2 \\ Presenting Complaint}

The patient was a 70-year-old man who came to the emergency department on March 20, 2002, complaining of heavy nasal bleeding for almost 3 days.

\section{Past Medical History}

History included severe ischemic cardiomyopathy with congestive heart failure and hypertension. An echocardiogram done on February 1, 2002, showed an ejection fraction of $20 \%$, and an apical mural thrombus was noticed in the left ventricle. The patient underwent anticoagulation therapy with warfarin on February 2, 2002. On February 28, he was seen in clinic for epistaxis. His $\mathrm{Hb}$ and $\mathrm{Hct}$ were $12.5 \mathrm{~g} / \mathrm{dL}$ and 0.409 , respectively, PT/aPTT were $26.9 / 50$ seconds, INR was 4.6 , and platelet count was normal. Medication and warfarin dosage adjustment was reviewed. Warning was given for uncontrolled bleeding and patient was advised to come back in a week for repeat INR. The patient did not return to clinic; instead he went to the emergency department on March 9 for protracted nasal bleeding that was not controlled by pressure. The patient was admitted to control the nasal bleeding. On this admission, his $\mathrm{Hb}$ and Hct were $11.1 \mathrm{~g} / \mathrm{dL}$ and 0.355 , respectively, PT/aPTT were $35.7 / 58$ seconds, INR was 7.8 , platelet count was $363,000 / \mathrm{cm}^{3}$, and liver function test was normal. His medication list included aspirin, isosorbide 
dinitrate, furosemide, potassium, quinapril, and digoxin. Vitamin K was administered, and the patient was discharged on a reduced dose of warfarin and advised to stop taking aspirin. Follow-up INR results were 2.6 and 1.3 on March 10 and 11, respectively. The patient did not keep his follow-up appointment scheduled for March 15 for a repeat check of his PT and INR. He presented to the emergency department on March 17, when his INR was found to be 9.4. Nasal packing was placed, warfarin was held, and patient was advised to follow up with outpatient clinic.

He presented to the outpatient clinic on March 20 , again with complaints of continued nasal bleeding that was profuse and unable to be stopped even with the packing in place. At 4:00 PM on March 20, his PT/aPTT were $37.4 / 72$ seconds and INR was 8.8. The patient was transferred to the emergency department for admission. An otolaryngologist saw the patient in the emergency department; the nose packing was replaced and $10 \mathrm{mg}$ of vitamin $\mathrm{K}$ was administered. A repeat test at 12:45 AM on March 21 showed that the PT/aPTT were 39.8/70 seconds, respectively, INR was $10, \mathrm{Hb} / \mathrm{Hct}$ were 10.2 $\mathrm{g} / \mathrm{dL}$ and 0.345 , and platelet count was $273,000 /$ $\mathrm{cm}^{3}$. On March 21 at 3:50 PM, PT/aPTT were shown to be higher at $47.5 / 80$ seconds, respectively, INR was $14.2, \mathrm{Hb} / \mathrm{Hct}$ were $10.1 \mathrm{~g} / \mathrm{dL}$ and 0.331 , respectively, and platelet count was $260,000 /$ $\mathrm{cm}^{3}$. Despite the administration of vitamin $\mathrm{K}$ again, the patient continued to bleed profusely, soaking the nasal packing. The on-call hematologist was consulted. Fresh frozen plasma (FFP) was considered but was not given to the patient because of his severe heart failure and the large volume of FFP required to correct his coagulopathy. Instead, rF.VIIa was considered as an alternative means to control the bleeding. Fibrinogen level was $483 \mathrm{mg}$ / dL. At 10:15 PM, I dose of rF.VIIa was given at 90 $\mu \mathrm{g} / \mathrm{kg}$ of body weight. Repeat PT/aPTT at 10:45 PM were $13.5 / 40$ seconds, respectively. $\mathrm{Hb} / \mathrm{Hct}$ were $10.1 \mathrm{~g} / \mathrm{dL} / 0.331$ with no changes in platelet level. For the next 3 days, his INR remained in the normal range. The nasal bleeding stopped, and the patient maintained hemostasis for the rest of his admission and was discharged on March 24. Patient was not placed on warfarin because of noncompliance. The patient remains stable with no further nasal bleeding. His last INR, from August 28, 2002, was 1.3 .

\section{Discussion}

Despite the different underlying causes of bleeding in the cases above, both responded to the administration of rF.VIIa. In liver cirrhosis thrombocytopenia, abnormal coagulation time and hyperfibrinolysis are present. At the end stage of liver disease, there is a decreased production of coagulation factors, factor VII being one of the most pronounced. This is reflected indirectly by the prolongation of prothrombin time. Methods of treating bleeding episodes in patients with cirrhosis currently include FFP, vitamin K, and desmopressin. Although FFP is the mainstay for treating coagulopathy, it does have serious side effects, such as paradoxical worsening of portal pressures, leading to further bleeding. Infection is also a potential risk because FFP is derived from human donors. Finally, administering FFP actually does not correct the underlying cause of the coagulopathy. Use of rF.VIIa can provide a new means of treating bleeding in cirrhotic patients. In our experience, rF.VIIa was very effective in controlling the continued bleeding caused by the scalp injury and there was no recurrence of the bleed. In circumstances in which all means to control the bleeding is exhausted, rF.VIIa may be tried.

The second case presented above was challenging. FFP could not be effectively used because of the sheer volume required to correct his coagulopathy caused by the severe heart failure. Patients treated with warfarin have low levels of functional vitamin $\mathrm{K}$-dependent coagulation factors. Administering vitamin $\mathrm{K}$ can reverse the effect of oral anticoagulation, but the reversal does not take place immediately. Thus, vitamin $\mathrm{K}$ will not be of help in the short term with severe bleedings. Coagulation factors can be administered in the form of FFP or clotting factor concentrate. FFP is inferior compared with CFC for reversal of coagulopathy, ${ }^{7}$ but potential thromboembolic side effects have been reported for CFC. $^{8}$ Recombinant F.VIIa can be used to control bleeding in patients on warfarin. The rationale for the success lies in the fact that F.VII has the shortest half-life of all vitamin Kdependent clotting factors, ${ }^{9}$ and it is quickly reduced when patients take warfarin. rF.VII helps in restoring the coagulation cascade rapidly.

\section{Conclusion}

Recombinant activated factor VII was initially developed for patients with hemophilia. The use has 
expanded to control bleeding in other clinical scenarios. It has been noted to have no side effects, and no reports of activation of the coagulation system have been reported. Cost should be taken into consideration because the price of 1.2-, 2.4-, and 4.8-mg doses are approximately $\$ 900, \$ 1900$, and $\$ 4000$, respectively. Judicious use of the medication should be undertaken so that it is used only when other means of reversing life-threatening coagulopathy fail. Further studies are needed to provide data to justify broadening the indications to use r.FVIIa in situations in which bleeding cannot be controlled by present modalities.

\section{References}

1. Vlot AJ, Ton E, Mackaay AJC, Kramer MHH, Gaillard CA. Treatment of a severely bleeding patient without preexisting coagulopathy with activated recombinant factor VII. Am J Med 2000;108:421-42.

2. White B, McHale J, Ravi N, et al. Successful use of recombinant FVIIa (NovoSeven) in the management of intractable post-surgical intrabdominal hemorrhage [letter] Br J Haematol 1999;107:677-67.

3. Jeffers L, Bernstein DE, Erhardsten E, et al. The use of recombinant factor VIIa in laparoscopy liver biopsy: a pilot trial [abstract]. Gastroentrology 1998; 114:L0275.

4. Martinowitz U, Kenet G, Segal E, et al. Recombinant activated factor VII for adjunctive hemorrhage control in trauma. J Trauma 2001;51:431-8.

5. O'Neill PA, Bluth M, Gloster ES, et al. Successful use of recombinant activated factor VII for traumaassociated hemorrhage in a patient without preexisting coagulopathy. J Trauma 2002;52:400-5.

6. Berntorp E. Recombinant FVIIa in the treatment of warfarin bleeding. Semin Thromb Hemost 2000;26: 433-5.

7. Makris M, Greaves M, Phillips WS, et al. Emergency oral anticoagulant reversal: the relative efficacy of infusions of fresh frozen plasma and clotting factor concentrate on correction of the coagulopathy. Thromb Haemost 1997;77:477-0.

8. Lusher JM. Factor IX concentrates. In: Forbes CD, Aledrt L, Madhok R, editors. Haemophilia. London: Chapman \& Hall Medical; 1997. p. 203-11.

9. vanDam-Nieras MC, Hemker HC. Half-life time and control frequency of vitamin $\mathrm{K}$ dependent coagulation factors. Theoretical considerations on the place of factor VII in the control of oral anticoagulation therapy. Haemostasis 1983;13:201-8. 